\title{
The Riemann zeta distribution
}

\author{
GWO DONG LIN $^{1}$ and CHIN-YUAN HU ${ }^{2}$ \\ ${ }^{1}$ Institute of Statistical Science, Academia Sinica, Taipei 11529, Taiwan, Republic of China. \\ E-mail: gdlin@stat.sinica.edu.tw \\ ${ }^{2}$ Department of Business Education, National Changhua University of Education, Changhua \\ 50058, Taiwan, Republic of China
}

Let $\zeta$ be the Riemann zeta function. Khinchine (1938) proved that the function $f_{\sigma}(t)=$ $\zeta(\sigma+\mathrm{i} t) / \zeta(\sigma)$, where $\sigma>1$ and $t$ is real, is an infinitely divisible characteristic function. We investigate further the fundamental properties of the corresponding distribution of $f_{\sigma}$, the Riemann zeta distribution, including its support and unimodality. In particular, the Riemann zeta random variable is represented as a linear function of infinitely many independent geometric random variables. To extend Khinchine's result, we construct the Dirichlet-type characteristic functions of discrete distributions and provide a sufficient condition for the infinite divisibility of these characteristic functions. By way of applications, we give probabilistic proofs for some identities in number theory, including a new identity for the reciprocal of the Riemann zeta function.

Keywords: completely multiplicative function; Dirichlet series; geometric distribution; infinite divisibility; Jordan totient function; Liouville function; Mangoldt function; Möbius function; Poisson distribution; Riemann zeta distribution; Riemann zeta function

\section{Introduction}

Consider the Riemann zeta function defined by $\zeta(z)=\sum_{n=1}^{\infty} n^{-z}$, where $z=\sigma+\mathrm{i} t, \sigma>1$ and $t$ is real. Khinchine (1938, p. 35) proved that for every $\sigma>1$, the normalized function

$$
f_{\sigma}(t)=\frac{\zeta(\sigma+\mathrm{i} t)}{\zeta(\sigma)}, \quad t \in \mathbb{R}
$$

is an infinitely divisible characteristic function (see also Gnedenko and Kolmogorov 1968, pp. 75-76). Surprisingly, few properties of $f_{\sigma}$ have been developed since 1938, as remarked by Chung (2001, p. 259): so far as known, this famous relationship between two 'big names' has produced no important issue.

For convenience the corresponding distribution $F_{\sigma}$ of $f_{\sigma}$ will be called the Riemann zeta distribution with parameter $\sigma$. In the next section we shall investigate the fundamental properties of $F_{\sigma}$, including its support and unimodality. In particular, we show that: (i) $F_{\sigma}$ is a discrete distribution with support $\{-\log n\}_{n=1}^{\infty}$; (ii) the saltus (jump) of $F_{\sigma}$ at the point $-\log n$ is $n^{-\sigma} / \zeta(\sigma)$; and (iii) the Riemann zeta random variable can be represented as a linear function of infinitely many independent geometric random variables.

More generally, we shall begin with the Dirichlet series which is an extension of the Riemann zeta function, and construct the Dirichlet-type characteristic functions of discrete distributions, comparing them with the Pólya-type characteristic functions of absolutely 
continuous distributions. Further, we prove that the Dirichlet-type characteristic function is infinitely divisible if the coefficient (as an arithmetical function) in the Dirichlet series is completely multiplicative. By way of applications, in Section 3 we shall give the probabilistic proofs for some identities in number theory, including a new identity for the reciprocal of the Riemann zeta function. A number of remarks are made in Section 4.

\section{Dirichlet-type characteristic functions}

In this section we begin with the Dirichlet series $D(z)=\sum_{n=1}^{\infty} c(n) / n^{z}$ with coefficients $c(n)$ non-negative and not all zero. Denote by $\sigma_{a}$ the abscissa of absolute convergence of $D(z)$. (This implies that the Dirichlet series $D(z)$ converges uniformly on every compact subset of the half-plane $\mathcal{K}_{a}=\left\{z: \operatorname{Re} z=\sigma>\sigma_{a}\right\}$; see, for example, Apostol 1976, p. 235.) For each $\sigma>\sigma_{a}$, define the normalized function

$$
\mathscr{D}_{\sigma}(t)=\frac{D(\sigma+\mathrm{i} t)}{D(\sigma)} \equiv \sum_{n=1}^{\infty} \frac{c_{*}(n)}{n^{\sigma+\mathrm{i} t}}, \quad \text { for } t \in \mathbb{R},
$$

where $c_{*}(n)=c(n) / D(\sigma)$. It will be seen that $\mathscr{D}_{\sigma}$ reduces to $f_{\sigma}$ if the coefficient $c(n)=1$ for each $n \in \mathbb{N} \equiv\{1,2, \ldots\}$. We shall show that the function $\mathscr{D}_{\sigma}$ is a bona fide characteristic function (cf) and that it is infinitely divisible if the coefficient $c(n)$ is completely multiplicative. The function $\mathscr{D}_{\sigma}$ will be called a cf of Dirichlet type. For convenience, let us define the random variable $W_{\sigma, D}$ corresponding to $D(z)$ by

$$
P\left(W_{\sigma, D}=n\right)=\frac{c(n) n^{-\sigma}}{D(\sigma)}, \quad \text { for } n \in \mathbb{N},
$$

where $\sigma>\sigma_{a}$ and $c(n), n \in \mathbb{N}$, are the coefficients in the Dirichlet series $D(z)$. Then we have the following result, in which $D^{(m)}$ denotes the $m$ th derivative of $D$.

Theorem 1. Let $D(z)$ be a Dirichlet series with coefficients $c(n)$ non-negative and not all zero. Let $\sigma_{a}$ be the abscissa of absolute convergence of $D(z)$ and $\sigma>\sigma_{a}$. Further, let $X_{\sigma, D}=-\log W_{\sigma, D}$, where $W_{\sigma, D}$ is the random variable defined in (3). Then

(a) the cf of $X_{\sigma, D}$ is $\mathscr{D}_{\sigma}$ given in (2);

(b) the mth moment $\mathrm{E}\left(X_{\sigma, D}^{m}\right)=D^{(m)}(\sigma) / D(\sigma)<\infty$ for each $m \in \mathbb{N}$.

Proof. By the definition of $X_{\sigma, D}$, its moment generating function has the explicit form $\mathrm{E}\left(\mathrm{e}^{t X_{\sigma, D}}\right)=D(\sigma+t) / D(\sigma)<\infty$ for $t>\sigma_{a}-\sigma$. Since $\sigma_{a}-\sigma<0$, the required results follow immediately.

Pólya's condition, which is very easy to apply, permits us to construct cfs of absolutely continuous distributions (see, for example, Lukacs 1970, p. 83). However, Theorem 1 above, which is also easy to apply, permits us to construct $\mathrm{cfs}$ of discrete distributions. For example, we now list seven more cfs involving the Riemann zeta function $\zeta$. For 
$z=\sigma+\mathrm{i} t, \sigma>1$ and $t \in \mathbb{R}$, let $D(z)$ be one of the following Dirichlet series with nonnegative coefficients:

$$
\frac{\zeta(z)}{\zeta(2 z)}, \quad \frac{\zeta^{2}(z)}{\zeta(2 z)}, \quad \frac{\zeta^{3}(z)}{\zeta(2 z)}, \quad \frac{\zeta^{3}(z)}{\zeta(3 z)}, \quad \frac{\zeta^{4}(z)}{\zeta(2 z)}, \quad \frac{\zeta(z) \zeta(2 z) \zeta(3 z)}{\zeta(6 z)}, \quad(-1)^{m} \zeta^{(m)}(z)
$$

(see Apostol 1976, pp. 241 and 247). Then, according to Theorem 1, the function $\mathscr{D}_{\sigma}(t)=D(\sigma+\mathrm{i} t) / D(\sigma), t \in \mathbb{R}$, is a cf.

Recall that the $\mathrm{cf} f_{\sigma}$ defined in (1) is infinitely divisible. It is natural to ask, if $\mathscr{D}_{\sigma}$ is a Dirichlet-type cf, under what conditions it is true that $\mathscr{D}_{\sigma}$ is still infinitely divisible. The surprisingly neat answer is that $\mathscr{D}_{\sigma}$ is infinitely divisible if the coefficient $c(n)$ in the Dirichlet series $D(z)$ is completely multiplicative, that is, if $c(n)$ satisfies the condition $c(m n)=c(m) c(n)$ for all positive integers $m$ and $n$. We first note that for the constant function $D(z)=1$ (with $c(1)=1$ and $c(n)=0$ for $n \geqslant 2$ ), the $\operatorname{cf} \mathscr{D}_{\sigma}=D$ and is infinitely divisible. For other cases, we state the results as follows.

Theorem 2. Let $D(z)$ be a Dirichlet series with completely multiplicative non-negative coefficients and $c(n)>0$ for some $n \geqslant 2$. Let $\sigma_{a}$ be the abscissa of absolute convergence of $D(z)$ and $\sigma>\sigma_{a}$. Then the function $\mathscr{D}_{\sigma}$ in (2) is an infinitely divisible $c f$.

Proof. Due to the completely multiplicative coefficients $c(n) \geqslant 0$, we can write

$$
D(\sigma+\mathrm{i} t)=\exp \left(\sum_{n=2}^{\infty} \frac{c(n) \Lambda(n)}{\log n} n^{-(\sigma+\mathrm{i} t)}\right) \equiv \exp \left(D^{*}(\sigma+\mathrm{i} t)\right), \quad \text { for } t \in \mathbb{R},
$$

where $\Lambda$ is the Mangoldt function (see Apostol 1976, p. 239). Therefore the function $\mathscr{D}_{\sigma}$ is of the form $\mathscr{D}_{\sigma}(t)=\exp \left(q\left\{\mathscr{D}_{\sigma}^{*}(t)-1\right\}\right)$ for $t \in \mathbb{R}$, in which $q=\log D(\sigma)=D^{*}(\sigma)>0$ and $\mathscr{D}_{\sigma}^{*}(t)=D^{*}(\sigma+\mathrm{i} t) / D^{*}(\sigma), t \in \mathbb{R}$, is a cf according to Theorem 1 . This implies that $\mathscr{D}_{\sigma}$ is an infinitely divisible cf (see Lukacs 1970, p. 111).

To guarantee the infinite divisibility of the $\mathrm{cf} \mathscr{D}_{\sigma}$, it is possible to assume other conditions instead of the completely multiplicative condition on $c(n)$. For details, see Remark 1 in Section 4. On the other hand, the infinite divisibility of $\mathscr{D}_{\sigma}$ suggests that we write $\mathscr{D}_{\sigma}$ as the limit of a sequence of finite products of Poisson-type cfs. To do this we need some notation. For each integer $n \geqslant 2$, define $U_{n}$ to be a Poisson random variable with parameter $\lambda_{n} \equiv c(n) \Lambda(n) /\left\{(\log n) n^{\sigma}\right\}$, where $c(n)$ and $\Lambda(n)$ are given in (4). That is,

$$
P\left(U_{n}=m\right)=\mathrm{e}^{-\lambda_{n}} \frac{\lambda_{n}^{m}}{m !}, \quad \text { for } m \in \mathbb{N}_{0} \equiv\{0,1,2, \ldots\},
$$

where $U_{n}=0$ almost surely if $\lambda_{n}=0$. Then we have the following interesting result, in which $\stackrel{d}{=}$ denotes equality in distribution.

Theorem 3. In addition to the conditions of Theorem 2, let $\left\{U_{n}\right\}_{n=2}^{\infty}$ be a sequence of independent Poisson random variables defined in (5). Assume, further, that $X_{\sigma, D}$ is a random 
variable with cf $\mathscr{D}_{\sigma}$. Then $X_{\sigma, D} \stackrel{d}{=} \sum_{n=2}^{\infty}(-\log n) U_{n}$ (that is, $X_{\sigma, D}$ can be represented as a linear function of infinitely many independent Poisson random variables).

Proof. Write $X_{n}=-(\log n) U_{n}$ for all $n \geqslant 2$. Then the cf of $X_{n}$ is $f_{X_{n}}(t)=$ $\mathrm{E}\left\{\exp \left(\mathrm{i} t(-\log n) U_{n}\right)\right\}=\exp \left(\lambda_{n}\left(n^{-\mathrm{i} t}-1\right)\right)$ for $t \in \mathbb{R}$. In the notation of the proof of Theorem 2, we have

$$
\prod_{n=2}^{\infty} f_{X_{n}}(t)=\exp \left(\sum_{n=2}^{\infty} \lambda_{n}\left(n^{-\mathrm{i} t}-1\right)\right)=\exp \left(q\left\{\mathscr{D}_{\sigma}^{*}(t)-1\right\}\right)=\mathscr{D}_{\sigma}(t), \quad \text { for } t \in \mathbb{R} .
$$

This implies that the series $\sum_{n=2}^{\infty} X_{n}$ of independent random variables converges almost surely (see, for example, Loève 1977, p. 263) and that $X_{\sigma, D} \stackrel{d}{=} \sum_{n=2}^{\infty} X_{n}$.

Given a Dirichlet series $D(z)$ with completely multiplicative non-negative coefficients and $c(n)>0$ for some $n \geqslant 2$, Theorems $1-3$ together assert that $W_{\sigma, D} \stackrel{d}{=} \prod_{n=2}^{\infty} n^{U_{n}}$ for each $\sigma>\sigma_{a}$. In the next theorem we shall further prove that if $c(p)<p^{\sigma}$ for each prime $p$, $W_{\sigma, D}$ has a factorization into prime factors with random exponents (a somewhat surprising property). To state the result we need some more notation. Given $\sigma>\sigma_{a}$, prime $p$ and $u_{p}=c(p) p^{-\sigma} \in[0,1)$, define the geometric random variable $N_{u_{p}}$ with parameter $u_{p}$ by

$$
P\left(N_{u_{p}}=n\right)=u_{p}^{n}\left(1-u_{p}\right), \quad \text { for } n \in \mathbb{N}_{0},
$$

where $N_{u_{p}}=0$ almost surely if $u_{p}=0$. Then we have the following striking result.

Theorem 4. Let $D(z)$ be a Dirichlet series with completely multiplicative non-negative coefficients and $c(n)>0$ for some $n \geqslant 2$. Let $\sigma_{a}$ be the abscissa of absolute convergence of $D(z)$ and let $\sigma>\sigma_{a}$ such that $u_{p} \equiv c(p) p^{-\sigma}<1$ for each prime $p$. Assume, further, that $X_{\sigma, D}=-\log W_{\sigma, D}$, where $W_{\sigma, D}$ is defined in (3), and that $\left\{N_{u_{p}}\right\}$ is a sequence of independent geometric random variables defined in (6). Then

(a) $X_{\sigma, D} \stackrel{d}{=} \sum_{p}(-\log p) N_{u_{p}}$, where $p$ runs over all primes;

(b) $W_{\sigma, D} \stackrel{d}{=} \prod_{p} p^{N_{u_{p}}}$ (a factorization of $W_{\sigma, D}$ into prime factors with random exponents).

Proof. Since the coefficient $c(n)$ is completely multiplicative, we have the identity

$$
D(z)=\sum_{n=1}^{\infty} \frac{c(n)}{n^{z}}=\prod_{p} \frac{1}{1-c(p) p^{-z}}, \quad \text { for } z=\sigma+\mathrm{i} t, \sigma>\sigma_{a}, t \in \mathbb{R}
$$

(Euler product formula: see Apostol 1976, p. 231). This, together with Theorem 1, implies that for each $\sigma>\sigma_{a}$, the $\mathrm{cf}$ of $X_{\sigma, D}$ can be written as

$$
\mathscr{D}_{\sigma}(t)=\frac{D(\sigma+\mathrm{i} t)}{D(\sigma)}=\prod_{p} \frac{1-c(p) p^{-\sigma}}{1-c(p) p^{-(\sigma+\mathrm{i} t)}}=\prod_{p} \frac{1-u_{p}}{1-u_{p} p^{-\mathrm{i} t}} \equiv \prod_{p} h_{p}(t), \quad \text { for } t \in \mathbb{R},
$$

where $p$ runs over all primes. Note that the factor $h_{p}$ in (7) is the infinitely divisible cf of $(-\log p) N_{u_{p}}$ (see Lukacs 1970, pp. 113 and 320). Equality (7) then implies that the series 
$\sum_{p}(-\log p) N_{u_{p}}$ converges almost surely and hence part (a) holds. Part (b) follows immediately from part (a).

A non-trivial arithmetical function $c(n)$ satisfying the conditions of Theorem 4 is given in Remark 2 below. Finally, we return to the case of Riemann zeta distribution. For Riemann zeta function $\zeta$, the random variables $W_{\sigma, D}$ and $N_{u_{p}}$ reduce respectively to $W_{\sigma}$ and $N_{p^{-\sigma}}$ with probabilities

$$
\begin{gathered}
P\left(W_{\sigma}=n\right)=\frac{n^{-\sigma}}{\zeta(\sigma)}, \quad \text { for } n \in \mathbb{N}, \\
P\left(N_{p^{-\sigma}}=n\right)=p^{-n \sigma}\left(1-p^{-\sigma}\right), \quad \text { for } n \in \mathbb{N}_{0} .
\end{gathered}
$$

Combining the above results, we describe the Riemann zeta distribution $F_{\sigma}$ as follows.

Corollary 1. Let $\sigma>1$ and let $X_{\sigma}$ be a random variable obeying the Riemann zeta distribution $F_{\sigma}$. Assume, further, that $W_{\sigma}$ is a random variable defined in (8) and that $\left\{N_{p^{-\sigma}}\right\}$ is a sequence of independent geometric random variables defined in (9). Then:

(a) $X_{\sigma} \stackrel{d}{=}-\log W_{\sigma} \stackrel{d}{=} \sum_{p}(-\log p) N_{p^{-\sigma}}$, where $p$ runs over all primes.

(b) $F_{\sigma}$ is infinitely divisible.

(c) $F_{\sigma}$ is a discrete distribution with support $\{-\log n\}_{n=1}^{\infty}$, and is unimodal with vertex at zero. Specifically, $P\left(X_{\sigma}=-\log n\right)=n^{-\sigma} / \zeta(\sigma)$ for each positive integer $n$.

(d) $\mathrm{E}\left(\exp \left(-t X_{\sigma}\right)\right)=\mathrm{E}\left(W_{\sigma}^{t}\right)=\zeta(\sigma-t) / \zeta(\sigma)<\infty$ for $t<\sigma-1$.

(e) $\mathrm{E}\left(X_{\sigma}^{m}\right)=\left((-1)^{m} / \zeta(\sigma)\right) \sum_{n=1}^{\infty}(\log n)^{m} / n^{\sigma}=\zeta^{(m)}(\sigma) / \zeta(\sigma)$ for each positive integer $m$.

\section{Applications to analytic number theory}

As pointed out in the Preface to Patterson (1995), the Riemann zeta function $\zeta$ is a meromorphic function whose properties can on the one hand be investigated by the techniques of complex analysis, and on the other yield different theorems concerning the integers; it is this connection between the continuous and the discrete that is so wonderful. For example, by Fourier theory, we have $\zeta(2)=\pi^{2} / 6$, a remarkable formula due to Leonard Euler. Actually, Euler proved much more, namely that for each integer $n>0, \zeta(2 n) / \pi^{2 n}$ is rational and related to the Bernoulli numbers. The evaluation of $\zeta(2 n)$ later came to play a fundamental role in the theory of $p$-adic zeta functions and Iwasawa theory (Patterson 1995, pp. 2-4). In this section, using the probabilistic approach, we shall derive some identities in order better to understand the Riemann zeta function. Two inequalities for $\zeta$ are given in the Remark 6 below.

The discrete random variable $W_{\sigma, D}$ defined in (3) plays an important role in the previous section. Its special case $W_{\sigma}$, defined in (8), will be used in the following to obtain our results in analytic number theory. For convenience, some required notions and results from number theory are given in the Appendix. We first propose an arithmetical function of $W_{\sigma}$ to link $W_{\sigma}$ and the Dirichlet series. More results through other arithmetical functions will be given later. 
Theorem 5. Let $D(z)$ be a Dirichlet series with coefficients $c(n)$ non-negative and not all zero, and converging absolutely for $\operatorname{Re} z=\sigma>\sigma_{a}$. If $A(n)=\sum_{d \mid n} c(d)$ for $n \in \mathbb{N}$, then $\mathrm{E}\left(A\left(W_{\sigma}\right)\right)=D(\sigma)$ for $\sigma>\max \left\{1, \sigma_{a}\right\}$.

Proof. First, for $\sigma>\max \left\{1, \sigma_{a}\right\}$, write

$$
\begin{aligned}
\mathrm{E}\left(A\left(W_{\sigma}\right)\right) & =\sum_{n=1}^{\infty} P\left(W_{\sigma}=n\right) A(n)=\sum_{n=1}^{\infty} \frac{n^{-\sigma}}{\zeta(\sigma)} A(n) \\
& =\frac{1}{\zeta(\sigma)} \sum_{n=1}^{\infty} \frac{A(n)}{n^{\sigma}}=\sum_{n=1}^{\infty} \frac{\mu(n)}{n^{\sigma}} \sum_{n=1}^{\infty} \frac{A(n)}{n^{\sigma}}=\sum_{n=1}^{\infty} \frac{(\mu * A)(n)}{n^{\sigma}},
\end{aligned}
$$

in which $\mu$ denotes the Möbius function and the symbol $*$ denotes the Dirichlet convolution. Then applying the Möbius inverse formula, we obtain that $\mathrm{E}\left(A\left(W_{\sigma}\right)\right)=\sum_{n=1}^{\infty} c(n) / n^{\sigma}$ $=D(\sigma)$, as desired.

Corollary 2. Assume $\sigma>1$ and integers $m \geqslant 1$. Then

(a) $\mathrm{E}\left(\tau\left(W_{\sigma}\right)\right)=\zeta(\sigma)$, where $\tau(n)$ is the number of divisors of $n$;

(b) $\mathrm{E}\left(\sum_{d \mid W_{\sigma}}(\log d)^{m}\right)=\sum_{n=1}^{\infty}(\log n)^{m} / n^{\sigma}=(-1)^{m} \xi^{(m)}(\sigma)$.

Proof. Take the Dirichlet series $D(z)=\zeta(z)=\sum_{n=1}^{\infty} 1 / n^{z}$ for part (a), and $D(z)=$ $\sum_{n=1}^{\infty}(\log n)^{m} / n^{z}$ for part (b). Then Theorem 5 applies.

In the next theorem we shall give probabilistic proofs for some identities connecting the Riemann zeta function $\zeta$ and the Mangoldt function $\Lambda$ as well as the Jordan totient function $J_{z}$. The latter is a generalization of the Euler totient function $\varphi$. Recall that $J_{1}(n)=\varphi(n)$ is defined to be the number of positive integers not exceeding $n$ which are relatively prime to $n$. In general, we have, for complex numbers $z \neq 0$,

$$
J_{z}(n)= \begin{cases}1 & \text { for } n=1, \\ n^{z} \prod_{p \mid n}\left(1-p^{-z}\right)=\sum_{d \mid n} \mu(d)\left(\frac{n}{d}\right)^{z} & \text { for } n \geqslant 2,\end{cases}
$$

in which $\mu$ denotes the Möbius function and the last equality is due to the lemma below. (See Apostol 1976, p. 48, for the special case $z=k \in \mathbb{N}$.) From now on, let $n=\prod_{j=1}^{k} p_{j}^{\alpha_{j}}$ denote the unique factorization of $n(\geqslant 2)$ into prime factors (with exponents $\alpha_{j} \geqslant 1$ ).

Lemma. (a) Assume that $A: \mathbb{N} \rightarrow \mathbb{C}$ is a multiplicative function with complex values (that is, $A(m n)=A(m) A(n)$ if $(m, n)=1)$. Then the Dirichlet convolution

$$
(\mu * A)(n)=\prod_{j=1}^{k}\left\{A\left(p_{j}^{\alpha_{j}}\right)-A\left(p_{j}^{\alpha_{j}-1}\right)\right\}, \quad \text { for } n=\prod_{j=1}^{k} p_{j}^{\alpha_{j}} \geqslant 2 .
$$

(b) If, in addition, $A$ is completely multiplicative and never vanishes, then 


$$
(\mu * A)(n)=A(n) \prod_{p \mid n}\{1-1 / A(p)\}, \quad \text { for } n \geqslant 2 .
$$

Proof. (a) Since $A$ is multiplicative, so is $\mu * A$ (see Apostol 1976, p. 35). Further, for prime $p$ and for integer $\alpha \geqslant 1$, we have $(\mu * A)\left(p^{\alpha}\right)=\mu(1) A\left(p^{\alpha}\right)+\mu(p) A\left(p^{\alpha-1}\right)=A\left(p^{\alpha}\right)-$ $A\left(p^{\alpha-1}\right)$. This implies result (11).

(b) Suppose that $A$ is completely multiplicative and never vanishes. Then we have $A\left(p^{\alpha}\right)-A\left(p^{\alpha-1}\right)=(A(p))^{\alpha}-(A(p))^{\alpha-1}=(A(p))^{\alpha}(1-1 / A(p))$. This, together with part (a), implies (12).

Theorem 6. (a) $\sum_{n=1}^{\infty} \Lambda(n) / n^{\sigma}=-\zeta^{\prime}(\sigma) / \zeta(\sigma)=\mathrm{E}\left(\log W_{\sigma}\right)$, for $\sigma>1$.

(b) $\sum_{n=1}^{\infty}(\Lambda(n) \log n) / n^{\sigma}=\operatorname{var}\left(\log W_{\sigma}\right)=\operatorname{var}\left(X_{\sigma}\right)$, for $\sigma>1$.

(c) $\sum_{n=1}^{\infty} J_{t}(n) / n^{\sigma}=\zeta(\sigma-t) / \zeta(\sigma)=\mathrm{E}\left(W_{\sigma}^{t}\right)$, for $t \in \mathbb{R}$ and $\sigma>\max \{1, t+1\}$.

Proof. (a) Set the function

$$
A(n)=\sum_{d \mid n} \Lambda(d)=\log n, \quad \text { for } n \in \mathbb{N}
$$

(see Apostol 1976, p. 32, for the second equality). Then, for $\sigma>1$, applying Theorem 5 to the Dirichlet series $D(z)=\sum_{n=1}^{\infty} \Lambda(n) / n^{z}$ yields

$$
\mathrm{E}\left(A\left(W_{\sigma}\right)\right)=\mathrm{E}\left(\sum_{d \mid W_{\sigma}} \Lambda(d)\right)=\sum_{n=1}^{\infty} \frac{\Lambda(n)}{n^{\sigma}} .
$$

On the other hand, we have $\mathrm{E}\left(\log W_{\sigma}\right)=-\zeta^{\prime}(\sigma) / \zeta(\sigma)$ according to Corollary 1(e). This, together with (13) and (14), implies the required result.

(b) Set the function $A(n)=(\log n)^{2} \equiv \log ^{2} n$ for $n \in \mathbb{N}$. Then proceeding as in the proof of Theorem 5 yields

$$
\mathrm{E}\left(\log ^{2} W_{\sigma}\right)=\sum_{n=1}^{\infty} \frac{\left(\mu * \log ^{2}\right)(n)}{n^{\sigma}}=\sum_{n=1}^{\infty} \frac{\Lambda(n) \log n}{n^{\sigma}}+\sum_{n=1}^{\infty} \frac{(\Lambda * \Lambda)(n)}{n^{\sigma}}, \quad \text { for } \sigma>1,
$$

the second equality following from the Selberg identity. This, together with part (a), implies the required result:

$$
\operatorname{var}\left(\log W_{\sigma}\right)=\mathrm{E}\left(\log ^{2} W_{\sigma}\right)-\left(\mathrm{E}\left(\log W_{\sigma}\right)\right)^{2}=\sum_{n=1}^{\infty} \frac{\Lambda(n) \log n}{n^{\sigma}} .
$$

(c) For each fixed $t \in \mathbb{R}$, set the function $A(n)=n^{t}, n \in \mathbb{N}$. Then we have

$$
\mathrm{E}\left(W_{\sigma}^{t}\right)=\mathrm{E}\left(A\left(W_{\sigma}\right)\right)=\sum_{n=1}^{\infty} \frac{(\mu * A)(n)}{n^{\sigma}}=\sum_{n=1}^{\infty} \frac{J_{t}(n)}{n^{\sigma}}, \quad \text { for } \sigma>\max \{1, t+1\},
$$

the last equality following from (10). Combining (15) with Corollary 1(d), we obtain the required result. 
In Theorem 5 we propose an arithmetical function of $W_{\sigma}$, denoted by $A\left(W_{\sigma}\right)$, to link $W_{\sigma}$ and the Dirichlet series. To obtain more applications of $W_{\sigma}$, we shall first consider the general arithmetical function $A: \mathbb{N} \rightarrow \mathbb{R}$, and then prove that the of of $\log A\left(W_{\sigma}\right)$ is infinitely divisible if $A$ is positive and completely multiplicative. In order to represent the mean and variance of $\log A\left(W_{\sigma}\right)$, we need a generalization of the Mangoldt function: for positive $A$, define $M_{A}(n)=\log A(p)$ if $n=p^{m}$ for some prime $p$ and some integer $m \geqslant 1$, and $M_{A}(n)=0$ otherwise. For the special case $A(n)=n, M_{A}$ reduces to the Mangoldt function $\Lambda$.

Theorem 7. Let $\sigma>1$ and let $A: \mathbb{N} \rightarrow \mathbb{R}$ be an arithmetical function. Then we have the following:

(a) The cf of $A\left(W_{\sigma}\right)$ is of the form $f_{\sigma, A}(t) \equiv \mathrm{E}\left(\mathrm{e}^{\mathrm{i} t A\left(W_{\sigma}\right)}\right)=\sum_{n=1}^{\infty}\left(\mu * \mathrm{e}^{\mathrm{i} t A}\right)(n) / n^{\sigma} \quad$ for $t \in \mathbb{R}$, where $\left(\mu * \mathrm{e}^{\mathrm{i} t A}\right)(n)=\sum_{d \mid n} \mu(d) \mathrm{e}^{\mathrm{i} t A(n / d)}$. Moreover, the mth moment $\mathrm{E}\left(A^{m}\left(W_{\sigma}\right)\right)=\sum_{n=1}^{\infty}\left(\mu * A^{m}\right)(n) / n^{\sigma}$ if it exists and is finite.

(b) The random variable $\log A\left(W_{\sigma}\right)$ has an infinitely divisible of if $A$ is positive and completely multiplicative. Moreover, $\mathrm{E}\left(\log A\left(W_{\sigma}\right)\right)=\sum_{n=1}^{\infty} M_{A}(n) / n^{\sigma}$ and $\operatorname{var}\left(\log A\left(W_{\sigma}\right)\right)=\sum_{n=1}^{\infty}\left\{M_{A}(n) \log A(n)\right\} / n^{\sigma}$ if they exist and are finite.

Proof. (a) The proof of the first identity for the $\mathrm{cf} f_{\sigma, A}$ is similar to that of Theorem 5 and is omitted. As for the moment identity, suppose that the $m$ th moment of $A\left(W_{\sigma}\right)$ exists and is finite. Then we have $f_{\sigma, A}^{(m)}(0)=\mathrm{i}^{m} \sum_{n=1}^{\infty}\left(\mu * A^{m}\right)(n) / n^{\sigma}$ and hence $\mathrm{E}\left(A^{m}\left(W_{\sigma}\right)\right)=$ $\mathrm{i}^{-m} f_{\sigma, A}^{(m)}(0)=\sum_{n=1}^{\infty}\left(\mu * A^{m}\right)(n) / n^{\sigma}$, as desired.

(b) Suppose that $A$ is positive and completely multiplicative. Then by Corollary 1(a), we have $A\left(W_{\sigma}\right) \stackrel{d}{=} A\left(\prod_{p} p^{N_{p^{-\sigma}}}\right)=\prod_{p}(A(p))^{N_{p^{-\sigma}}}$, where $p$ runs over all primes and $\left\{N_{p^{-\sigma}}\right\}$ is a sequence of independent geometric random variables defined in (9). This implies that $\log A\left(W_{\sigma}\right) \stackrel{d}{=} \sum_{p}(\log A(p)) N_{p^{-\sigma}}$. Therefore the cf of $\log A\left(W_{\sigma}\right)$ is the limit of a sequence of infinitely divisible cfs, and hence also infinitely divisible. On the other hand, if the mean of $\log A\left(W_{\sigma}\right)$ exists and is finite, we have

$$
\begin{aligned}
\mathrm{E}\left(\log A\left(W_{\sigma}\right)\right) & =\sum_{p}(\log A(p)) \mathrm{E}\left(N_{p^{-\sigma}}\right)=\sum_{p}(\log A(p)) \frac{p^{-\sigma}}{1-p^{-\sigma}} \\
& =\sum_{p} \sum_{m=1}^{\infty} \frac{\log A(p)}{\left(p^{m}\right)^{\sigma}}=\sum_{n=1}^{\infty} \frac{M_{A}(n)}{n^{\sigma}} .
\end{aligned}
$$

Further, if the second moment of $\log A\left(W_{\sigma}\right)$ is finite, we have

$$
\begin{aligned}
\operatorname{var}\left(\log A\left(W_{\sigma}\right)\right) & =\sum_{p}(\log A(p))^{2} \operatorname{var}\left(N_{p^{-\sigma}}\right)=\sum_{p}(\log A(p))^{2} \frac{p^{-\sigma}}{\left(1-p^{-\sigma}\right)^{2}} \\
& =\sum_{p} \sum_{m=1}^{\infty}(\log A(p))^{2} m\left(p^{-\sigma}\right)^{m}=\sum_{p} \sum_{m=1}^{\infty} \frac{\log A(p) \log A\left(p^{m}\right)}{\left(p^{m}\right)^{\sigma}} \\
& =\sum_{n=1}^{\infty} \frac{M_{A}(n) \log A(n)}{n^{\sigma}} .
\end{aligned}
$$


The penultimate equality is due to the completely multiplicative property of $A$.

Finally, we apply Theorem 7 to derive two identities for the reciprocal of the Riemann zeta function. To do this we need the arithmetical functions $\beta$ and $\gamma$ defined below. For $n \geqslant 1$, (i) $\beta(n)=m$ if $n=p_{1} \ldots p_{m}\left(p_{m+1} \ldots p_{k}\right)^{2}$, and $\beta(n)=0$ otherwise; (ii) $\gamma(n)=k$ if $n=\left(p_{1} \ldots p_{k}\right)^{2}$, and $\gamma(n)=0$ otherwise. Further, define the sign function $\operatorname{sgn} x=0$ or $x /|x|$ according to whether $x=0$ or $x \neq 0$. To the best of the authors' knowledge, the interesting identity in part (b) below appears for the first time. (Recall the classical result that $(\zeta(\sigma))^{-1}=\sum_{n=1}^{\infty} \mu(n) / n^{\sigma}, \sigma>1$.)

Theorem 8. Assume $\sigma>1$. Then

(a) $(\zeta(\sigma))^{-2}=1+\sum_{n=2}^{\infty}\left\{(-2)^{\beta(n)} \operatorname{sgn} \beta(n)\right\} / n^{\sigma}=\mathrm{E}\left(\mu\left(W_{\sigma}\right)\right)$;

(b) $(\zeta(2 \sigma))^{-1}=1+\sum_{n=2}^{\infty}\left\{(-1)^{\gamma(n)} \operatorname{sgn} \gamma(n)\right\} / n^{\sigma}=\mathrm{E}\left(\mu^{2}\left(W_{\sigma}\right)\right)$.

Proof. It will be seen that the $m$ th moment $\mathrm{E}\left(\mu^{m}\left(W_{\sigma}\right)\right)$ equals $\mathrm{E}\left(\mu\left(W_{\sigma}\right)\right)$ or $\mathrm{E}\left(\mu^{2}\left(W_{\sigma}\right)\right)$ according to whether $m$ is odd or even.

(a) For each prime $p$, set $c_{p}(\alpha)=\mu\left(p^{\alpha}\right)-\mu\left(p^{\alpha-1}\right), \alpha \in \mathbb{N}$. Then $c_{p}(1)=-2, c_{p}(2)=1$, and $c_{p}(\alpha)=0$ for $\alpha \geqslant 3$. Since $\mu$ is multiplicative, we have, by the lemma above,

$$
(\mu * \mu)(n)=\prod_{j=1}^{k} c_{p_{j}}\left(\alpha_{j}\right)=(-2)^{\beta(n)} \operatorname{sgn} \beta(n), \quad \text { for } n=\prod_{j=1}^{k} p_{j}^{\alpha_{j}} \geqslant 2 .
$$

It then follows from Theorem 7(a) that

$$
\mathrm{E}\left(\mu\left(W_{\sigma}\right)\right)=\sum_{n=1}^{\infty} \frac{(\mu * \mu)(n)}{n^{\sigma}}=1+\sum_{n=2}^{\infty} \frac{(-2)^{\beta(n)} \operatorname{sgn} \beta(n)}{n^{\sigma}} .
$$

On the other hand, we have, by the definition of $W_{\sigma}$,

$$
\mathrm{E}\left(\mu\left(W_{\sigma}\right)\right)=\frac{1}{\zeta(\sigma)} \sum_{n=1}^{\infty} \frac{\mu(n)}{n^{\sigma}}=\frac{1}{\zeta^{2}(\sigma)} .
$$

Combining this with (16) yields the required result.

(b) For each prime $p$, set $c_{p}^{*}(\alpha)=\mu^{2}\left(p^{\alpha}\right)-\mu^{2}\left(p^{\alpha-1}\right), \alpha \in \mathbb{N}$. Then $c_{p}^{*}(\alpha)=-1$ or $c_{p}^{*}(\alpha)=0$ according to whether $\alpha=2$ or $\alpha \neq 2$. Since $\mu^{2}$ is multiplicative, we have

$$
\left(\mu * \mu^{2}\right)(n)=\prod_{j=1}^{k} c_{p_{j}}^{*}\left(\alpha_{j}\right)=(-1)^{\gamma(n)} \operatorname{sgn} \gamma(n), \quad \text { for } n=\prod_{j=1}^{k} p_{j}^{\alpha_{j}} \geqslant 2 .
$$

Again from Theorem 7(a), it follows that

$$
\mathrm{E}\left(\mu^{2}\left(W_{\sigma}\right)\right)=\sum_{n=1}^{\infty} \frac{\left(\mu * \mu^{2}\right)(n)}{n^{\sigma}}=1+\sum_{n=2}^{\infty} \frac{(-1)^{\gamma(n)} \operatorname{sgn} \gamma(n)}{n^{\sigma}} .
$$

On the other hand, we have 


$$
\mathrm{E}\left(\mu^{2}\left(W_{\sigma}\right)\right)=\frac{1}{\zeta(\sigma)} \sum_{n=1}^{\infty} \frac{\mu^{2}(n)}{n^{\sigma}}=\frac{1}{\zeta(\sigma)} \frac{\zeta(\sigma)}{\zeta(2 \sigma)}=\frac{1}{\zeta(2 \sigma)}
$$

(see Apostol 1976, p. 241, for the second equality). Combining this with (17) yields the required result.

\section{Remarks}

Remark 1. In Theorem 2, the completely multiplicative condition on $c(n)$ is not a necessary condition for the infinite divisibility of the $\mathrm{cf} \mathscr{D}_{\sigma}$. To see this, note first that multiplying the function $c(n)$ in $D(z)$ by a positive constant keeps $\mathscr{D}_{\sigma}$ unchanged, but the resulting function, say $d \cdot c(n)$, is not always completely multiplicative. Further, to guarantee the infinite divisibility of $\mathscr{D}_{\sigma}$, we may assume other conditions on $c(n)$. For example, in addition to the conditions of Theorem 1, assume that (i) $c(1)>0$; (ii) $D(z) \neq 0$ for $\operatorname{Re} z=\sigma>\sigma_{0}\left(\sigma_{0}\right.$ being a fixed number $\left.\geqslant \sigma_{a}\right)$; and (iii) $\left(c^{\prime} * c^{-1}\right)(n) \geqslant 0$ for $n \geqslant 2$, where $c^{\prime}(n) \equiv(\log n) c(n)$ and $c^{-1}$ is the Dirichlet inverse of $c$. Then applying Theorem 11.14 of Apostol (1976) and proceeding as in Theorem 3 (but instead taking the parameter $\lambda_{n}=\left(c^{\prime} * c^{-1}\right)(n) /\left\{(\log n) n^{\sigma}\right\}$ in (5)), we conclude that, for $\sigma>\sigma_{0}$, the $\operatorname{cf} \mathscr{D}_{\sigma}$ is infinitely divisible.

Remark 2. We shall now introduce a non-trivial function $c(n)$ which satisfies the conditions of Theorem 4. For complex numbers $z$, define the generalized Liouville function $\lambda_{z}$ by

$$
\lambda_{z}(n)= \begin{cases}1 & \text { for } n=1, \\ z^{\alpha_{1}+\ldots+\alpha_{k}} & \text { for } n=\prod_{j=1}^{k} p_{j}^{\alpha_{j}} \geqslant 2 .\end{cases}
$$

Then it will be seen that $\lambda_{z}$ is completely multiplicative. Moreover, for fixed $\sigma_{0}>1$ and $a \in\left(0,2^{\sigma_{0}-1}\right)$, set the function $c(n)=\lambda_{a}(n)>0, \quad n \in \mathbb{N}$. Then $D(z)=\sum_{n=1}^{\infty} c(n) / n^{z}$ converges absolutely for $\operatorname{Re} z=\sigma>\sigma_{0}$, and $c(p)=a<p^{\sigma}$ for each prime $p$. Therefore $c(n)$ satisfies the conditions of Theorem 4.

Remark 3. Note that Theorem 7 also generalizes Khinchine's (1938) result in a sense, but the approach is different from Theorem 2. To see this, let $A(n)=n$ for $n \in \mathbb{N}$; then Theorem 7(b) asserts that the random variable $\log W_{\sigma}$ has an infinitely divisible cf. On the other hand, letting $A\left(W_{\sigma}\right)=\log W_{\sigma} \equiv Y_{\sigma} \quad$ in Theorem $7\left(\right.$ a) yields $\mathrm{E}\left(\mathrm{e}^{\mathrm{i} t Y_{\sigma}}\right)=\sum_{n=1}^{\infty} J_{\mathrm{i} t}(n) / n^{\sigma}=$ $\zeta(\sigma-\mathrm{i} t) / \zeta(\sigma)$ for $t \in \mathbb{R}$ and $\sigma>1$, where $J_{\mathrm{i} t}$ is the Jordan totient function defined in (10).

Remark 4. Consider the Liouville function $\lambda=\lambda_{-1}$ defined in Remark 2. We now calculate all moments of $\lambda\left(W_{\sigma}\right)$. Note first that $\lambda$ is completely multiplicative and never vanishes. Using the lemma above, we have

$$
(\mu * \lambda)(n)=\lambda(n) \prod_{p \mid n}\left(1-\frac{1}{\lambda(p)}\right)=\lambda(n) \prod_{p \mid n} 2=\lambda(n) 2^{\nu(n)},
$$


where $v(n)$ denotes the number of distinct prime factors of $n$. Theorem 7(a) then implies that

$$
\mathrm{E}\left(\lambda\left(W_{\sigma}\right)\right)=\sum_{n=1}^{\infty} \frac{(\mu * \lambda)(n)}{n^{\sigma}}=\sum_{n=1}^{\infty} \frac{\lambda(n) 2^{\nu(n)}}{n^{\sigma}}=\frac{\xi(2 \sigma)}{\xi^{2}(\sigma)}, \quad \text { for } \sigma>1
$$

(see Apostol 1976, p. 247, for the last equality). Next, for $n \in \mathbb{N}$, we have $\lambda^{m}(n)$ equal to 1 or $\lambda(n)$ according to whether $m$ is even or odd. Therefore the $m$ th moment $\mathrm{E}\left(\lambda^{m}\left(W_{\sigma}\right)\right)$ is 1 or $\zeta(2 \sigma) / \zeta^{2}(\sigma)$ according to whether $m$ is even or odd. Moreover, $\operatorname{var}\left(\lambda\left(W_{\sigma}\right)\right)=$ $1-\zeta^{2}(2 \sigma) / \zeta^{4}(\sigma)$ for $\sigma>1$.

Remark 5. For $a>0$, the generalized Liouville function $\lambda_{a}$ is positive and completely multiplicative. By Theorem 7(b), the random variable $\log \lambda_{a}\left(W_{\sigma}\right)$ has an infinitely divisible cf, which, however, is not of Dirichlet type. On the other hand, for real $a \neq 0,1$, proceeding as in Remark 4, we extend identity (18) to the following:

$$
\mathrm{E}\left(\lambda_{a}\left(W_{\sigma}\right)\right)=\sum_{n=1}^{\infty} \frac{\lambda_{a}(n)\left(1-a^{-1}\right)^{v(n)}}{n^{\sigma}}=\frac{1}{\zeta(\sigma)} \sum_{n=1}^{\infty} \frac{\lambda_{a}(n)}{n^{\sigma}}, \quad \text { for } \sigma>\max \left\{1,1+\frac{\log |a|}{\log 2}\right\} .
$$

The crucial point of the proof is that $\left|\lambda_{a}(n)\right| \leqslant n^{(\log |a|) / \log 2}$ for $n \geqslant 1$.

Remark 6. Finally, we give two inequalities for the Riemann zeta function. From Corollary $1(\mathrm{e})$ it follows that for $\sigma>1, \zeta^{\prime \prime}(\sigma) \zeta(\sigma)>\left(\zeta^{\prime}(\sigma)\right)^{2}$ because $\operatorname{var}\left(X_{\sigma}\right)>0$. Similarly, by Corollary 1(d), $\zeta(\sigma-2) \xi(\sigma)>\xi^{2}(\sigma-1)$ for $\sigma>3$. More generally, using Lyapunov's inequality, we conclude that the function $h(t)=(\zeta(\sigma-t) / \zeta(\sigma))^{1 / t}$ is strictly increasing in $t \in(0, \sigma-1)$.

\section{Appendix}

The following notions and results from number theory (see Apostol 1976) are needed in Sections 3 and 4.

- Möbius function $\mu$. The Möbius function $\mu$ is defined as follows: $\mu(1)=1$; for $n=\prod_{j=1}^{k} p_{j}^{\alpha_{j}} \geqslant 2$ (the unique factorization of $n$ into prime factors), $\mu(n)=(-1)^{k}$ if $\alpha_{1}=\alpha_{2}=\ldots=\alpha_{k}=1$, and $\mu(n)=0$ otherwise.

- Möbius inverse formula. The equation $f(n)=\sum_{d \mid n} g(d)$ implies $g(n)=$ $\sum_{d \mid n} f(d) \mu(n / d)$, and vice versa.

- Dirichlet convolution. If $f$ and $g$ are two arithmetical functions, their Dirichlet convolution is defined to be the function $(f * g)(n)=\sum_{d \mid n} f(d) g(n / d), n=1,2, \ldots$

- Dirichlet inverse function. If $f$ is an arithmetical function with $f(1) \neq 0$, then there is a unique function $f^{-1}$, called the Dirichlet inverse of $f$, such that $f * f^{-1}=$ $f^{-1} * f=I$, where $I$ is the identity function defined by $I(n)=1$ if $n=1$, and $I(n)=0$ otherwise.

- Mangoldt function $\Lambda$. For every positive integer $n$, define $\Lambda(n)=\log p$ if $n=p^{m}$ for some prime $p$ and some $m \geqslant 1$, and $\Lambda(n)=0$ otherwise. 
- Selberg identity. For $n \geqslant 1$ we have

$$
\Lambda(n) \log n+\sum_{d \mid n} \Lambda(d) \Lambda\left(\frac{n}{d}\right)=\sum_{d \mid n} \mu(d) \log ^{2} \frac{n}{d} .
$$

\section{Acknowledgements}

The authors would like to thank the Professor Ole E. Barndorff-Nielsen, an associate editor and two referees for their helpful comments and suggestions, which have greatly improved the presentation of the paper.

\section{References}

Apostol, T.M. (1976) Introduction to Analytic Number Theory. New York: Springer-Verlag.

Chung, K.L. (2001) A Course in Probability Theory, 3rd edition. New York: Academic Press.

Gnedenko, B.V. and Kolmogorov, A.N. (1968) Limit Distributions for Sums of Independent Random Variables, transl. K.L. Chung. Reading, MA: Addison-Wesley.

Khinchine, A.Ya. (1938) Limit Theorems for Sums of Independent Random Variables (in Russian). Moscow and Leningrad: GONTI.

Loève, M. (1977) Probability Theory I, 4th edition. New York: Springer-Verlag.

Lukacs, E. (1970) Characteristic Functions, 2nd edition. New York: Hafner.

Patterson, S.J. (1995) An Introduction to the Theory of the Riemann Zeta-Function. Cambridge: Cambridge University Press.

Received October 1999 and revised June 2001 